\title{
A GENERALIZATION OF ZUBOV'S METHOD TO PERTURBED SYSTEMS*
}

\author{
FABIO CAMILLI ${ }^{\dagger}$, LARS GRÜNE ${ }^{\ddagger}$, AND FABIAN WIRTH ${ }^{\S}$
}

\begin{abstract}
A generalization of Zubov's theorem on representing the domain of attraction via the solution of a suitable partial differential equation is presented for the case of perturbed systems with a singular fixed point. For the construction it is necessary to consider solutions in the viscosity sense. As a consequence maximal robust Lyapunov functions can be characterized as viscosity solutions.
\end{abstract}

1. Introduction. The domain of attraction of an asymptotically stable fixed point has been one of the central objects in the study of continuous dynamical systems. In the late 1960s there was a particular surge of activity with a number of papers by Coleman [8], Wilson [24], Bhatia [6] analyzing properties of the domains. One of the celebrated results of that era was what came to be known as Zubov's method [25] which asserts that the domain of attraction of an asymptotically stable fixed point $x^{*}$ of

$$
\dot{x}=f(x), \quad x \in \mathbb{R}^{n}
$$

may be characterized by solutions $v$ of the partial differential equation

$$
D v(x) \cdot f(x)=-h(x)(1-v(x)) \sqrt{1+\|f(x)\|^{2}} .
$$

Namely, under suitable assumptions on $h$, the set $v^{-1}([0,1))$ is equal to the domain of attraction. These results are presented in several books, see [10] or [13]. For the case of real-analytic systems a constructive procedure is presented in [10] that allows for the approximation of the domain of attraction. This method was extended and simplified in [23], where again a constructive approach for the case of analytic systems is presented. The construction was extended to the case of asymptotically stable periodic orbits in [2].

In recent years much effort has been devoted to the development of numerical methods for the approximation of domains of attractions. Zubov's method also lends itself to the construction of such schemes, see [23], [12] and the paper [1] which considers a particular application.

In this paper our aim is to generalize Zubov's basic result by incorporating perturbations into the setup. That is we consider systems of the form

$$
\dot{x}=f(x, a),
$$

with the property that the fixed point (which we take to be zero) is not perturbed under all perturbations. Under a local stability assumption, which guarantees that it is reasonable to consider domains of attraction we are interested in the set of points

\footnotetext{
*Research supported by the TMR Networks "Nonlinear Control Network" and "Viscosity Solutions and their applications" and DFG Priority Research Program "Ergodentheorie, Analysis und effiziente Simulation dynamischer Systeme"

${ }^{\dagger}$ Dip. di Energetica, Fac. di Ingegneria, Università de l’Aquila, 67040 Roio Poggio (AQ), Italy, camilli@axcasp. caspur.it

${ }^{\ddagger}$ Fachbereich Mathematik, J.W. Goethe-Universität, Postfach 1119 32, 60054 Frankfurt a.M., Germany, gruene@math.uni-frankfurt.de

$\S$ Zentrum für Technomathematik, Universität Bremen, 28334 Bremen, Germany, fabian@math.uni-bremen.de
} 
that is attracted to the fixed point regardless of the perturbation considered. This is what we call the robust domain of attraction. This subset of the domain of the unperturbed system $\dot{x}=f\left(x, a_{0}\right)$ is also studied in [17], [18], where in particular an approximation scheme for the robust domain of attraction is presented based on ideas of optimal control. In this paper we concentrate on proving an existence and uniqueness result for a Zubov type equation and examining properties of the solutions that can be obtained. Numerical aspects and actual examples are presented in [7].

In the following Section 2 we begin defining robust domains of attraction for the class of systems under consideration and state some fundamental properties. In the ensuing Section 3 we define the generalization of (1.1) suitable for our case and discuss the question of solvability of this equation. For this we turn to the methodology of viscosity solutions. We refer to [3] for an introduction to this theory in the context of optimal control. Using viscosity solutions we obtain an existence and uniqueness result for the generalized equation. In Sections 4 and 5 we note some properties of the constructed solutions. In particular, the solutions can be interpreted as robust Lyapunov functions for the perturbed system and via suitable choices of the parameters this Lyapunov function can be guaranteed to be globally Lipschitz, or smooth at least on subsets of the robust domain of attraction. Finally, in Section 6 we provide a simple example illustrating our results.

2. The robust domain of attraction. Let $\varphi\left(t, x_{0}, a\right)$ be the solution of

$$
\left\{\begin{array}{l}
\dot{x}(t)=f(x(t), a(t)), \quad t \in[0, \infty), \\
x(0)=x_{0}
\end{array}\right.
$$

where $a(\cdot) \in \mathcal{A}=L^{\infty}([0,+\infty), A)$ and $A$ is a compact subset of $\mathbb{R}^{m}$. Throughout the paper the map $f$ is taken to be continuous and bounded in $\mathbb{R}^{n} \times A$ and locally Lipschitz in $x$ uniformly in $a \in A$. Furthermore, we assume that the fixed point $x=0$ is singular, that is $f(0, a)=0$ for any $a \in A$.

We assume that the singular point 0 is uniformly locally exponentially stable for the system (2.1), i.e.

$$
\begin{aligned}
& \text { there exist constants } C, \sigma, r>0 \text { such that }\left\|\varphi\left(t, x_{0}, a\right)\right\| \leq C e^{-\sigma t}\left\|x_{0}\right\| \\
& \text { for any } x_{0} \in B(0, r) \text { and any } a \in \mathcal{A} \text {. }
\end{aligned}
$$

We now define the following sets which describe domains of attraction for the equilibrium $x=0$ of the system (2.1).

DeFinition 2.1. For the system (2.1) satisfying (H1) we define the robust domain of attraction as

$$
\mathcal{D}=\left\{x_{0} \in \mathbb{R}^{n}: \varphi\left(t, x_{0}, a\right) \rightarrow 0 \text { as } t \rightarrow+\infty \text { for any } a \in \mathcal{A}\right\},
$$

and the uniform robust domain of attraction by

$$
\mathcal{D}_{0}=\left\{\begin{array}{ll}
x_{0} \in \mathbb{R}^{n}: \begin{array}{l}
\text { there exists a function } \beta(t) \rightarrow 0 \text { as } t \rightarrow \infty \\
\text { s.th. }\left\|\varphi\left(t, x_{0}, a\right)\right\| \leq \beta(t) \text { for all } t>0, a \in \mathcal{A}
\end{array}
\end{array}\right\} .
$$

In order to obtain a different characterization of $\mathcal{D}_{0}$ we introduce the following "first hitting time" defined by $t(x, a):=\inf \{t>0: \varphi(t, x, a) \in B(0, r)\}$. Note that by the assumption on $B(0, r)$ there exists $T>0$ independent of $x$ and $a$ such that $\varphi(t, x, a) \in B(0, r)$ for any $t \geq t(x, a)+T$. 


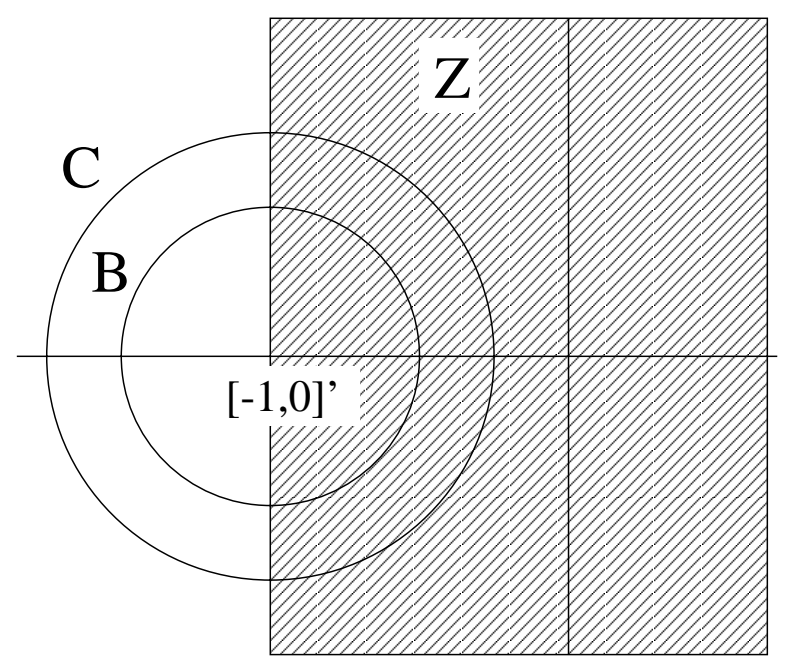

FiG. 2.1. Sketch for Example 2.1

Lemma 2.2. Assume (H1), then the robust domains of attraction $\mathcal{D}$ and $\mathcal{D}_{0}$ satisfy

$$
\begin{aligned}
\mathcal{D} & =\left\{x \in \mathbb{R}^{n}: t(x, a)<+\infty \text { for any } a \in \mathcal{A}\right\} . \\
\mathcal{D}_{0} & =\left\{x \in \mathbb{R}^{n}: \sup _{a \in \mathcal{A}}\{t(x, a)\}<+\infty\right\} .
\end{aligned}
$$

Proof. This is immediate from Definition 2.1.

Before we begin analyzing some of the properties of $\mathcal{D}$ and $\mathcal{D}_{0}$ let us give an example that shows that for general nonlinear systems they are different.

EXAMPLE 2.1. Let $n=2, y_{0}=[-1,0]^{\prime}$. We fix two discs around $y_{0}$ given by $B:=B\left(y_{0}, 1 / 2\right)$ and $C:=B\left(y_{0}, 3 / 4\right)$ and let $Z:=\left\{x=\left[x_{1}, x_{2}\right] \in \mathbb{R}^{2}: x_{1}>-1\right\}$, see Figure 2.1.

Fix a $C^{\infty}$ function $h: \mathbb{R}^{2} \rightarrow \mathbb{R}$ such that

$$
h \geq 0, \quad h_{\mid B} \equiv 1, \quad h_{\mid C^{c}} \equiv 0 .
$$

Fix any $f: \mathbb{R}^{2} \rightarrow \mathbb{R}^{2}$ such that $f_{\mid B} \equiv 0, f(0)=0$ and such that the set $Z \backslash$ cl $B$ is contained in the domain of attraction of $x^{*}=0$ for the system $\dot{x}=f(x)$. We may assume furthermore that for the first component function of $f$ (denoted by $f_{1}$ ) we have $f_{1}(x)>0$ on the annulus $C \backslash \mathrm{cl} B$. Now consider the system

$$
\dot{x}=f(x)+h(x)\left[\begin{array}{c}
\left(x_{1}+1\right)^{2}+x_{2}^{2}+1-a^{2} \\
a
\end{array}\right]=: g(x, a),
$$

where $a \in[-1,1]$. It is easy to see that for $x_{0} \in B$ it holds that the first component of the solution $\varphi_{1}\left(t, x_{0}, a\right)$ is strictly increasing as long as $\varphi\left(t, x_{0}, a\right) \in B$. We even have that for any $x_{0} \in B \cap \operatorname{cl} Z$ and any $a \in \mathcal{A}$ there is a time $T=T\left(x_{0}, a\right)$ such that $\varphi\left(T, x_{0}, a\right) \notin B$. Also by our assumption on $f, h$ and the construction of $g$ the first component of the solutions is strictly increasing on $C \backslash \mathrm{cl} B$. As a consequence $y_{0} \in \mathcal{D}$. On the other hand for $y_{0}$ we have that for any time $t>0$ and any $\varepsilon>0$ 
there is some $a \in \mathcal{A}$ with $\left\|y_{0}-\varphi\left(t, y_{0}, a\right)\right\|<\varepsilon$ by [11, Chap. 3,Theorem 6] as 0 is contained in the convex hull of $\left\{g\left(y_{0}, a\right): a \in A\right\}$. Hence, $t\left(y_{0}, a\right)$ is unbounded over $\mathcal{A}$ and so $y_{0} \notin \mathcal{D}_{0}$.

In the following proposition we present some relevant properties of the (uniform) robust domain of attraction. Several of these bear a striking resemblance to those of the domain of attraction of an asymptotically stable fixed point of a time-invariant system, compare [10, Chap. IV]. It will frequently be convenient to consider the reachable set at time $T$ from an initial condition $x_{0} \in \mathbb{R}^{n}$ defined by

$$
\mathcal{R}\left(x_{0}, T_{0}\right):=\left\{x \in \mathbb{R}^{n}: \exists 0 \leq t \leq T_{0}, a \in \mathcal{A} \text { such that } x=\varphi\left(t, x_{0}, a\right)\right\} .
$$

Note that by the boundedness of $f$ it is immediate that the reachable set from a bounded set of initial conditions $S$ given by

$$
\mathcal{R}(S, T):=\bigcup_{x \in S} \mathcal{R}(x, T)
$$

is bounded for any $T \geq 0$.

Proposition 2.3. Consider system (2.1) and assume (H1), then

(i) $\operatorname{cl} B(0, r) \subset \mathcal{D}_{0}$.

(ii) $\mathcal{D}_{0}$ is an open, connected, invariant set. $\mathcal{D}$ is a pathwise connected, invariant set.

(iii) $\sup _{a \in \mathcal{A}}\{t(x, a)\} \rightarrow+\infty$ for $x \rightarrow x_{0} \in \partial \mathcal{D}_{0}$ or $\|x\| \rightarrow \infty$.

(iv) $\mathcal{D} \subset \operatorname{cl} \mathcal{D}_{0}$

(v) $\operatorname{cl} \mathcal{D}_{0}=\operatorname{cl} \mathcal{D}$ is an invariant set.

(vi) $\mathcal{D}_{0}, \mathcal{D}$ are contractible to 0.

(vii) If for some $a_{0} \in A f\left(\cdot, a_{0}\right)$ is of class $C^{1}$, then $\mathcal{D}_{0}$ is $C^{1}$-diffeomorphic to $\mathbb{R}^{n}$.

(viii) If for every $x \in \partial \mathcal{D}_{0}$ there exists $a \in \mathcal{A}$ such that $\varphi(t, x, a) \in \partial \mathcal{D}_{0}$ for all $t \geq 0$ then $\mathcal{D}=\mathcal{D}_{0}$.

(ix) If for all $x \in \mathcal{D}$ the set $\{f(x, a): a \in \mathcal{A}\}$ is convex then $\mathcal{D}_{0}=\mathcal{D}$.

Proof.

(i) This is a consequence of the exponential bound in (H1), which can easily be shown to extend to $\operatorname{cl} B(0, r)$.

(ii) Let $x_{0} \in \mathcal{D}_{0}$ and $T_{0}=\sup _{a \in \mathcal{A}}\left\{t\left(x_{0}, a\right)\right\}$. Then there exists $T$ such that $\varphi\left(t, x_{0}, a\right) \in B(0, r / 2 C)$ for any $a \in \mathcal{A}, t \geq T$. Let $\delta$ be such that if $\left\|x_{0}-x\right\| \leq$ $\delta$, then $\left\|\varphi\left(t, x_{0}, a\right)-\varphi(t, x, a)\right\| \leq r / 2 C$ for any $t \leq T$ and any $a \in \mathcal{A}$. Then $\varphi(t, x, a) \in B(0, r)$ for $t \geq T$ and $a \in \mathcal{A}$. Therefore $x \in \mathcal{D}_{0}$ and it follows that $\mathcal{D}_{0}$ is open. By definition from each $x \in \mathcal{D}_{0}(x \in \mathcal{D})$ there exists a trajectory $\varphi(\cdot, x, a)$ entering $B(0, r)$. This shows connectedness. To prove invariance assume that for some $x \in \mathcal{D}_{0}, a_{1} \in \mathcal{A}$ there exists a $t>0$ such that $y:=\varphi\left(t, x, a_{1}\right) \notin \mathcal{D}_{0}$. This implies $\sup _{a \in \mathcal{A}}\{t(y, a)\}=\infty$. But clearly, $\sup _{a \in \mathcal{A}}\{t(x, a)\} \geq \sup _{a \in \mathcal{A}}\{t(y, a)\}$ contradicting the choice of $x$. A similar argument works for $\mathcal{D}$.

(iii) Let $x_{n} \rightarrow x_{0} \in \partial \mathcal{D}_{0}$ and set $T_{n}=\sup _{a \in \mathcal{A}}\left\{t\left(x_{n}, a\right)\right\}$. If we assume that $T_{n}$ is bounded and we take $r^{\prime}<r$, we can find $T$ such that, for any $n$, $\varphi\left(t, x_{n}, a\right) \in B\left(0, r^{\prime}\right)$ for any $t \geq T$ and for any $a \in \mathcal{A}$.

For any $\epsilon>0$, there exists $\delta>0$ such that if $\left\|x^{\prime}-x^{\prime \prime}\right\| \leq \delta, \| \varphi\left(t, x^{\prime}, a\right)-$ $\varphi\left(t, x^{\prime \prime}, a\right) \| \leq \epsilon$ for any $t \leq T$, for any any $a \in \mathcal{A}$. Thus, setting $\epsilon=r-r^{\prime}$ and choosing $n$ sufficiently large such that $\left\|x_{n}-x_{0}\right\| \leq \delta$ we obtain that $\varphi\left(t, x_{0}, a\right) \in B(0, r)$ for any $t \geq T$ and for any $a \in \mathcal{A}$. Hence $x_{0} \in \mathcal{D}_{0}$, a contradiction. The assertion is clear for $\left\|x_{n}\right\| \rightarrow \infty$, as our assumptions exclude solutions exploding in backward time. 
(iv) The statement follows from an application of [20, Lemma III.2], which states that if $x \in \mathcal{D} \backslash \mathcal{D}_{0}$, or equivalently, if $\sup _{a \in \mathcal{A}}\{t(x, a)\}=\infty$, while $t(x, a)<\infty$ for every $a \in \mathcal{A}$, then $x \in \partial \mathcal{D}$ as in every neighborhood of $x$ there exists a point $y$ and a control $a_{y}$ such that $t\left(y, a_{y}\right)=\infty$.

(v) If for some $x \in \operatorname{cl} \mathcal{D}_{0}$ and $a \in \mathcal{A}$ we have $\varphi(t, x, a) \notin \operatorname{cl} \mathcal{D}_{0}$ then by continuous dependence on initial conditions we have that $\mathcal{D}_{0}$ is not invariant, contradicting (i). The equality of the two sets is an immediate consequence of (iv).

(vi) This follows by regarding the flow of $\dot{x}=f\left(x, a_{0}\right)$ for some $a_{0} \in \mathcal{A}$.

(vii) In the proof we follow the outline given in [18]. Recall that a paracompact manifold $M$ with the property that every compact subset of $M$ has an open neighborhood that is diffeomorphic to $\mathbb{R}^{n}$ is itself diffeomorphic to $\mathbb{R}^{n}$, see [16, Lemma 3]. Let $K \subset \mathcal{D}_{0}$ be compact and consider a neighborhood $U$ of $K$ with $B(0, r) \subset U \subset \mathcal{D}_{0}$. Choose a relatively compact neighborhood $U_{2}$ of $K$ with $B(0, r / 2) \subset \operatorname{cl} U_{2} \subset U$ and fix a $C^{\infty}$ function $h: \mathbb{R}^{n} \rightarrow[0,1]$ with $h_{\mid U_{2}} \equiv 1$ and $h_{\mid U^{c}} \equiv 0$. Now consider the system

$$
\dot{x}=h(x) f\left(x, a_{0}\right),
$$

with associated flow $\psi(t, x)$. It is clear that for some $T$ large enough we have $K \subset \psi(-T, B(0, r / 4)) \subset U$. This proves the assertion as $\psi(-T, B(0, r / 4))$ is diffeomorphic to $B(0, r / 4)$ which is in turn diffeomorphic to $\mathbb{R}^{n}$.

(viii) By the pathwise connectedness of $\mathcal{D}$ we have that $\mathcal{D} \cap \partial \mathcal{D}_{0} \neq \emptyset$ if $\mathcal{D} \neq \mathcal{D}_{0}$. This contradicts our assumption.

(ix) Clearly we need only show $\mathcal{D} \subset \mathcal{D}_{0}$. Assume that $x \in \mathcal{D}$ and there exist sequences $a_{k} \in \mathcal{A}, T_{k} \rightarrow \infty$ such that $\left\|\varphi\left(T_{k}, x, a_{k}\right)\right\|>r>0$ for all $k \in \mathbb{N}$. By standard constructions there exists a subsequence (for which we use the index $k$ again) which converges uniformly on compact time intervals to a solution $y(t)$ of the differential inclusion

$$
\dot{y}(t) \in f(y(t), A) .
$$

Now by convexity of $f(y(t), A), t \geq 0$ and Filippov's lemma [14, p. 267] there exists a control $a \in \mathcal{A}$ such that $y(t)=\varphi(t, x, a)$. By assumption there exists a $t_{0}$ such that $\left\|\varphi\left(t_{0}, x, a\right)\right\|<r / C$. As $\varphi\left(t_{0}, x, a_{k}\right) \rightarrow \varphi\left(t_{0}, x, a\right)$ this implies for all $k$ large enough the inequality $\left\|\varphi\left(t, x, a_{k}\right)\right\|<r$ for $t \geq t_{0}$, a contradiction.

口

3. Zubov's method for robust domains of attraction. It is our aim to show that the appropriate generalization of Zubov's equation (1.1) is given by

$$
\inf _{a \in A}\{-D v(x) f(x, a)-(1-v(x)) g(x, a)\}=0 \quad x \in \mathbb{R}^{n} .
$$

In this section we show the existence of a unique solution under a suitable "boundary condition" in the equilibrium $x=0$. This solution will turn out to characterize the uniform robust domain of attraction $\mathcal{D}_{0}$. Before turning to this equation we introduce two optimal value functions and show certain properties of these functions. by

Consider the following nonnegative, extended value function $V: \mathbb{R}^{n} \rightarrow \mathbb{R} \cup\{+\infty\}$

$$
V(x)=\sup _{a \in \mathcal{A}} \int_{0}^{+\infty} g(\varphi(t, x, a), a(t)) d t
$$


and its transformation via the Kruzkov transform

$$
v(x)=1-e^{-V(x)} .
$$

The function $g: \mathbb{R}^{n} \times A \rightarrow \mathbb{R}$ is supposed to be continuous and satisfies

(i) For any $a \in A, g(0, a)=0$ and $g(x, a)>0$ for $x \neq 0$.

(ii) There exists a constant $g_{0}>0$ such that $\inf _{x \notin B(0, r), a \in A} g(x, a) \geq g_{0}$.

(iii) For every $R>0$ there exists a constant $L_{R}$ such that $\|g(x, a)-g(y, a)\| \leq L_{R}\|x-y\|$ for all $\|x\|,\|y\| \leq R$, and all $a \in A$.

Since $g$ is nonnegative it is immediate that $V(x) \geq 0$ and $v(x) \in[0,1]$ for all $x \in \mathbb{R}^{n}$. Furthermore, standard techniques from optimal control (see e.g. [3, Chapter III]) imply that $V$ and $v$ satisfy the dynamic programming principle, i.e. for each $t>0$ we have

$$
V(x)=\sup _{a \in \mathcal{A}}\left\{\int_{0}^{t} g(\varphi(\tau, x, a), a(\tau)) d \tau+V(\varphi(t, x, a))\right\}
$$

and

$$
v(x)=\sup _{a \in \mathcal{A}}\{(1-G(x, t, a))+G(x, t, a) v(\varphi(t, x, a))\}
$$

with

$$
G(x, t, a):=\exp \left(-\int_{0}^{t} g(\varphi(\tau, x, a), a(\tau)) d \tau\right)
$$

The relation between $V$ and $v$ is immediate; we have

$$
\begin{array}{ll}
V(x)=0 & \Leftrightarrow v(x)=0 . \\
V(x) \in(0,+\infty) & \Leftrightarrow v(x) \in(0,1) . \\
V(x)=+\infty & \Leftrightarrow v(x)=1 .
\end{array}
$$

In the next proposition we investigate the relation between $\mathcal{D}_{0}$ and $V$ (and thus also $v$ ), and the continuity of $V$ and $v$.

Proposition 3.1. Assume (H1), (H2). Then

(i) $V(x)<+\infty$ if and only if $x \in \mathcal{D}_{0}$.

(ii) $V(0)=0$ if and only if $x=0$.

(iii) $V$ is continuous on $\mathcal{D}_{0}$.

(iv) $V(x) \rightarrow+\infty$ for $x \rightarrow x_{0} \in \partial \mathcal{D}_{0}$ and for $\|x\| \rightarrow \infty$.

(v) $v(x)<1$ if and only if $x \in \mathcal{D}_{0}$.

(vi) $v(0)=0$ if and only if $x=0$.

(vii) $v$ is continuous on $\mathbb{R}^{n}$.

(viii) $v(x) \rightarrow 1$ for $x \rightarrow x_{0} \in \partial \mathcal{D}_{0}$ and for $\|x\| \rightarrow \infty$.

Proof. (i) To show that $V\left(x_{0}\right)<+\infty$ for $x_{0} \in \mathcal{D}_{0}$, observe that by Lemma 2.2 for each $x_{0} \in \mathcal{D}_{0}$ there exists $T_{0}>0$ such that $\varphi\left(t, x_{0}, a\right) \in B(0, r)$ for all $t \geq T_{0}$ and all $a \in \mathcal{A}$. Also the closure of the reachable set $\operatorname{cl} \mathcal{R}\left(x_{0}, T_{0}\right)$ is compact. Thus for any $a \in \mathcal{A}$

$$
\begin{aligned}
\int_{0}^{+\infty} g(\varphi(t), a(t)) d t & \leq \int_{0}^{T_{0}} g(\varphi(t), a(t)) d t+L_{r} \int_{T_{0}}^{+\infty}\|\varphi(t)\| d t \\
& \leq T_{0} \sup _{x \in \mathcal{R}\left(x_{0}, T_{0}\right), a \in A} g(x, a)+L_{r} C \int_{T_{0}}^{+\infty} e^{-\sigma t} r d t \leq \tilde{C}
\end{aligned}
$$


with $\tilde{C}$ independent of $a \in \mathcal{A}$ and therefore $V\left(x_{0}\right)<+\infty$.

Now let $x_{0} \notin \mathcal{D}_{0}$. Then there exists a sequence $a_{n} \in \mathcal{A}$ such that $t\left(x_{0}, a_{n}\right)$ tends to $\infty$. Then for any $n \in \mathbb{N}$

$$
\int_{0}^{+\infty} g\left(\varphi(t), a_{n}(t)\right) d t \geq \int_{0}^{t\left(x_{0}, a_{n}\right)} g\left(\varphi(t), a_{n}(t)\right) d t \geq g_{0} t\left(x_{0}, a_{n}\right),
$$

where $g_{0}>0$ is defined as in (H2) (ii). It follows that $V(x)=+\infty$.

(ii) Follows immediately from (3.1), (H2) (i), and $f(0, a)=0$.

(iii) Observe that

$$
\begin{aligned}
|V(x)-V(y)| & =\left|\sup _{a \in \mathcal{A}} \int_{0}^{+\infty} g(\varphi(t, x, a), a(t)) d t-\sup _{a \in \mathcal{A}} \int_{0}^{+\infty} g(\varphi(t, y, a), a(t)) d t\right| \\
& \leq \sup _{a \in \mathcal{A}} \int_{0}^{+\infty}|g(\varphi(t, x, a), a(t))-g(\varphi(t, y, a), a(t))| d t .
\end{aligned}
$$

We first prove that $V$ is continuous on $B(0, r / C)$.

Fix some $x_{0} \in B(0, r / C)$. Then (H1) and (H2) (iii) imply

$$
\begin{aligned}
\int_{0}^{+\infty} g\left(\varphi\left(t, x_{0}, a\right), a(t)\right) d t & \leq L_{r} \int_{0}^{+\infty}\left\|\varphi\left(t, x_{0}, a\right)\right\| d t \\
& \leq L_{r} C \int_{0}^{+\infty} e^{-\sigma t}\left\|x_{0}\right\| d t \leq C_{1}\left\|x_{0}\right\| .
\end{aligned}
$$

Fix $\epsilon>0$. From (H1) we can conclude that there exists $T>0$ such that $C_{1}\|\varphi(t, x, a)\| \leq \epsilon / 4$ for all $t \geq T$ and all $x \in B(0, r / C)$. Abbreviate $L=L_{r / C}$. Then by Lipschitz continuity of $f$ there exists a $\delta>0$ such that $\left\|\varphi\left(t, x_{0}, a\right)-\varphi\left(t, y_{0}, a\right)\right\|<$ $\epsilon /(2 L T)$ for all $t \in[0, T]$ and all $y_{0} \in B(0, r / C)$ with $\left\|x_{0}-y_{0}\right\|<\delta$.

Putting this together yields for every $a \in \mathcal{A}$

$$
\begin{aligned}
& \int_{0}^{+\infty}\left|g\left(\varphi\left(t, x_{0}, a\right), a(t)\right)-g\left(\varphi\left(t, y_{0}, a\right), a(t)\right)\right| d t \\
& \leq \int_{0}^{T} L\left\|\varphi\left(t, x_{0}, a\right)-\varphi\left(t, y_{0}, a\right)\right\| d t+C_{1}\left\|\varphi\left(T, x_{0}, a\right)\right\|+C_{1}\left\|\varphi\left(T, y_{0}, a\right)\right\| \\
& \leq \epsilon / 2+\epsilon / 4+\epsilon / 4 \leq \epsilon,
\end{aligned}
$$

which by (3.7) implies continuity.

For $x_{0} \in \mathcal{D}_{0}$ we can use openness of $\mathcal{D}_{0}$ in order to conclude that there exists an open neighborhood $N$ of $x_{0}$ and $T>0$ such that $\varphi\left(t, y_{0}, a\right) \in B(0, r / C)$ for all $y_{0} \in N$, all $a \in \mathcal{A}$ and all $t \geq T$. Thus (3.3) and the continuity on $B(0, r / C)$ imply continuity in $x_{0}$.

(iv) Follows immediately from Proposition 2.3 (iii) since $\mathcal{D}_{0}$ is open and $g(x) \geq$ $g_{0}>0$ for $x$ outside $B(0, r)$ as assumed in (H2).

(v) and (vi) follow immediately from (3.6), (i) and (ii), (vii) follows from (3.6), (iii) and (iv), and (viii) follows from (3.6) and (iv).

We now turn to the formulation of suitable partial differential equations for which $V$ and $v$ form solutions. Since in general these functions will not be differentiable we have to work with a more general solution concept, namely viscosity solutions.

Let us recall the definition of viscosity solutions (for more details about this theory we refer to [3]). 
Definition 3.2. Given an open subset $\Omega$ of $\mathbb{R}^{n}$ and a continuous function $H$ : $\Omega \times \mathbb{R} \times \mathbb{R}^{n} \rightarrow \mathbb{R}$, we say that a lower semicontinuous (l.s.c.) function $u: \Omega \rightarrow \mathbb{R}$ (resp. an upper semicontinuous (u.s.c.) function $v: \Omega \rightarrow \mathbb{R}$ ) is a viscosity supersolution (resp. subsolution) of the equation

$$
H(x, u, D u)=0 \quad x \in \Omega
$$

if for all $\phi \in C^{1}(\Omega)$ and $x \in \operatorname{argmin}_{\Omega}(u-\phi)$ (resp., $x \in \operatorname{argmax}_{\Omega}(v-\phi)$ ) we have

$$
H(x, u(x), D \phi(x)) \geq 0 \quad(\text { resp. }, H(x, v(x), D \phi(x)) \leq 0) .
$$

$A$ continuous function $u: \Omega \rightarrow \mathbb{R}$ is said to be a viscosity solution of (3.8) if $u$ is a viscosity supersolution and a viscosity subsolution of (3.8).

REMARK 3.1. It is not difficult to see (cf. [3, Lemma II.1.7]) that the set of derivatives $D \phi(x)$ for $x \in \operatorname{argmin}_{\Omega}(u-\phi)$ coincides with the set

$$
D^{-} u(x):=\left\{p \in \mathbb{R}^{n} \mid u(x)-u(y)-p(x-y) \geq-o(\|x-y\|) \text { for all } y \in \mathbb{R}^{n}\right\}
$$

and that the set of derivatives $D \phi(x)$ for $x \in \operatorname{argmax}_{\Omega}(v-\phi)$ equals

$$
\left.D^{+} v(x):=\left\{p \in \mathbb{R}^{n} \mid v(x)-v(y)-p(x-y) \leq o(\|x-y\|) \text { for all } y \in \mathbb{R}^{n}\right\}\right\} .
$$

Hence, one can alternatively define viscosity solutions via the sets $D^{-}$and $D^{+}$, the so called sub-and superdifferentials. Note that if a function $w: \Omega \rightarrow \mathbb{R}$ is differentiable in some $x \in \Omega$ the equality $D^{+} w(x)=D^{-} w(x)=\{D w(x)\}$ follows, hence for smooth functions viscosity solutions coincide with classical solutions.

Recalling that $V$ is locally bounded in $\mathcal{D}_{0}$, and $v$ is locally bounded on $\mathbb{R}^{n}$ the following proposition follows from an easy application of the dynamic programming principles (3.3) and (3.4), cp. [3, Chapter III].

Proposition 3.3. $V$ is a viscosity solution of

$$
\inf _{a \in A}\{-D V(x) f(x, a)-g(x, a)\}=0 \quad x \in \mathcal{D}_{0}
$$

and $v$ is a viscosity solution of

$$
\inf _{a \in A}\{-D v(x) f(x, a)-(1-v(x)) g(x, a)\}=0 \quad x \in \mathbb{R}^{n} .
$$

Observe that (3.10) is the straightforward generalization of the classical Zubov equation (1.1) [25] multiplied by -1 , which is necessary in order to obtain the proper sign for viscosity sub- and supersolutions. Equation (3.9), however, shows that also our "auxiliary function" $V$ can be characterized as the solution of a suitable PDE.

In order to get a uniqueness result we use the following super- and suboptimality principles. Our approach is closely related to that of Soravia [21, 22]; we quote the following result from [21].

TheOREM 3.4. ([21, Theorem 3.2 (i)]) Consider the equation

$$
\sup _{a \in A}\{-D u(x) f(x, a)-h(x, a)+k(x, a) u(x)\}=0 .
$$

Then if $u$ is an u.s.c. subsolution of (3.11) then it satisfies the lower optimality principle

$$
\begin{aligned}
u(x)=\inf _{a \in \mathcal{A}} \inf _{t \geq 0} & {\left[\int_{0}^{t} \exp \left(-\int_{0}^{s} k(\varphi(r), a(r)) d r\right) h(\varphi(s), a(s)) d s\right.} \\
& \left.+\exp \left(-\int_{0}^{t} k(\varphi(t), a(t)) d s\right) u(\varphi(t))\right]
\end{aligned}
$$


Recalling the definition of $G$ from (3.5), we see that this result has immediate applications for (3.9), (3.10).

PROPOSITION 3.5.

(i) Let $w$ be a l.s.c. supersolution of (3.10) in $\mathbb{R}^{n}$, then for any $x \in \mathbb{R}^{n}$

$$
w(x)=\sup _{a \in \mathcal{A}} \sup _{t \geq 0}\{(1-G(x, t, a))+G(x, t, a) w(\varphi(t))\} .
$$

(ii) Let $W$ be a l.s.c. supersolution of (3.9) in $\mathcal{D}_{0}$, then for any $x \in \mathcal{D}_{0}$

$$
W(x)=\sup _{a \in \mathcal{A}} \sup _{t \geq 0}\left\{\int_{0}^{t} g(\varphi(s), a(s)) d s+W(\varphi(t))\right\} .
$$

(iii) Let $u$ be an u.s.c. subsolution of $(3.10)$ in $\mathbb{R}^{n}$, and $\tilde{u}: \mathbb{R}^{n} \rightarrow \mathbb{R}$ be a continuous function with $u \leq \tilde{u}$. Then for any $x \in \mathbb{R}^{n}$ and any $T \geq 0$

$$
u(x) \leq \sup _{a \in \mathcal{A}} \inf _{t \in[0, T]}\{(1-G(x, t, a))+G(x, t, a) \tilde{u}(\varphi(t))\} .
$$

(iv) Let $U$ be an u.s.c. subsolution of $(3.9)$ in $\mathcal{D}_{0}$, and $\tilde{U}: \mathcal{D}_{0} \rightarrow \mathbb{R}$ be a continuous function with $U \leq \tilde{U}$. Then for any $x \in \mathcal{D}_{0}$ and any $T \geq 0$

$$
U(x) \leq \sup _{a \in \mathcal{A}} \inf _{t \in[0, T]}\left\{\int_{0}^{t} g(\varphi(s), a(s)) d s+\tilde{U}(\varphi(t))\right\} .
$$

Proof. If $w$ is a l.s.c. supersolution of (3.10), then it follows by multiplication by -1 and an application of the definition, that $-w$ is an u.s.c. subsolution of

$$
\sup _{a \in A}\{-D u(x) f(x, a)+(1+u(x)) g(x, a)\}=0 \quad x \in \mathbb{R}^{n} .
$$

This implies that we can directly apply Theorem 3.4 for the special case $h \equiv-g, k \equiv g$ to obtain that $-w$ satisfies

$$
\begin{aligned}
-w(x)=\inf _{a \in \mathcal{A}} \inf _{t \geq 0} & {\left[-\int_{0}^{t} \exp \left(-\int_{0}^{s} g(\varphi(r), a(r)) d r\right) g(\varphi(s), a(s)) d s\right.} \\
& \left.-\exp \left(-\int_{0}^{t} g(\varphi(t), a(t)) d s\right) w(\varphi(t))\right]
\end{aligned}
$$

Now the assertion follows upon multiplication by -1 and using the fact that

$$
\int_{0}^{t} G(\varphi(s), s, a(s)) g(\varphi(s), a(s)) d s=1-G(x, t, a) .
$$

(ii) follows by insertion of $k \equiv 0, h \equiv-g$ in (3.11).

For the proof of (iii) we follow the ideas of [21] with minor modifications. Let $u: \mathbb{R}^{n} \rightarrow \mathbb{R}$ be an upper semicontinuous subsolution of (3.10), let $\tilde{u}: \mathbb{R}^{n} \rightarrow \mathbb{R}$ be a continuous function with $u \leq \tilde{u}$ and define $u^{*}:=-u$ and $\tilde{u}^{*}:=-\tilde{u}$. Again a straightforward verification of the definition shows that $u^{*}$ is a lower semicontinuous viscosity supersolution of

$$
\sup _{a \in A}\{-D w(x) f(x, a)+(1+w(x)) g(x, a)\}=0 \quad x \in \mathbb{R}^{n} .
$$


From this equation it is easy to see that the auxiliary function $\bar{u}: \mathbb{R}^{n+2} \rightarrow \mathbb{R}$ given by $\bar{u}(x, r, s)=e^{-s} u^{*}(x)+r$ is a lower semicontinuous supersolution of

$$
\sup _{a \in A}\left\{-e^{-s} D_{x} v(x, r, s) f(x, a)+D_{r} v(x, r, s) e^{-s} g(x, a)-D_{s} v(x, r, s) g(x, a)\right\}=0
$$

for $x \in \mathbb{R}^{n}, r, s \in \mathbb{R}$.

We now introduce a change of variables by choosing $\rho: \mathbb{R} \rightarrow \mathbb{R}_{+}$smooth, bounded and such that $0<\dot{\rho} \leq M$ and $\rho(s) \rightarrow 0$ as $s \rightarrow-\infty$. Now consider the function

$$
U(z)=U(x, r, s):=\rho(\bar{u}(x, r, s))=\rho\left(e^{-s} u^{*}(x)+r\right) .
$$

By the rules for changes of variables (cf. [3, Proposition II.2.5]) it can be shown that $U$ is a lower semicontinuous supersolution of

$$
\sup _{a \in A}\left\{-D_{z} u(z) F(z, a)\right\}=0, \quad z \in \mathbb{R}^{n+2},
$$

where the underlying dynamics is given by

$$
\dot{z}=\left(\begin{array}{c}
\dot{x} \\
\dot{r} \\
\dot{s}
\end{array}\right)=F(z(t), a(t))=\left(\begin{array}{c}
f(\varphi(t), a(t)) \\
-e^{-s(t)} g(\varphi(t), a(t)) \\
g(\varphi(t), a(t))
\end{array}\right)
$$

Note that the solution to this system corresponding to an initial value $z=(x, 0,0)$ is given by

$$
z(t)=\left[\varphi(t, x, a),(G(t, x, a)-1), \int_{0}^{t} g(\varphi(s), a(s)) d s\right]^{\prime} .
$$

In order to apply results from [21, Appendix] we need that $F$ satisfies a global Lipschitz condition. Since this is not true in general, we localize the problem by considering for $k \in \mathbb{N}$ the family of smooth functions $\zeta_{k}: \mathbb{R}^{n+2} \rightarrow \mathbb{R}$ with $0 \leq \zeta_{k} \leq 1$, $\zeta_{k} \equiv 1$ in $B(0, k) \subset \mathbb{R}^{n+2}, \zeta_{k} \equiv 0$ in $B(0, k+1)^{c},\left|D \zeta_{k}\right| \leq 2$, and setting $F_{k}=\zeta_{k} F$.

Then from (3.18) we can conclude that for each $k \in \mathbb{N}$ the function $U$ is also a supersolution of

$$
\sup _{a \in A}\left\{-D_{z} u(z) F_{k}(z, a)\right\}=0
$$

as the multiplication with the nonnegative function $\zeta_{k}$ does not affect the inequality, that a supersolution has to fulfill.

Now consider the continuous function $\phi: \mathbb{R}^{n+2} \rightarrow \mathbb{R}, \phi(z)=\phi(x, s, r), x \in$ $\mathbb{R}^{n}, s, r \in \mathbb{R}$

$$
\phi(z)=\rho\left(e^{-s} \tilde{u}^{*}(x)+r\right) .
$$

Since $U \geq 0$ (by the choice of $\rho$ ) we obtain for any fixed $\lambda>0$ that $U$ is also a supersolution of

$$
\lambda u+\min \left\{\sup _{a \in A}\left\{-D_{z} u(z) F_{k}(z, a)\right\}, u-(1+\lambda) \phi\right\}=0,
$$

This equation has a unique continuous viscosity solution and it can be shown $[21$, Appendix] that this solution is given by the value function

$$
V_{k}^{\lambda}(z):=\inf _{a \in \mathcal{A}} \sup _{t \geq 0} e^{-\lambda t} \phi\left(z_{k}(t)\right)
$$


where $z_{k}(\cdot)$ solves $\dot{z}_{k}(t)=F_{k}\left(z_{k}(t), a(t)\right), z_{k}(0)=z$. By the usual comparison theorem for semicontinuous viscosity solution (see e.g. [3, Chapter V]) we obtain $U \geq V_{k}^{\lambda}$ for each $\lambda>0$ and each $k \in \mathbb{N}$. Hence letting $\lambda \rightarrow 0$ yields for all $k \in \mathbb{N}$ and all $T>0$ the inequality

$$
\rho\left(e^{-s} u^{*}(x)+r\right)=U(z) \geq \inf _{a \in \mathcal{A}} \sup _{t \in[0, T]} \phi\left(z_{k}(t)\right) .
$$

By the boundedness of $f$ the reachable set $\mathcal{R}(x, T)$ is bounded for each $x \in \mathbb{R}^{n}, T>0$. Hence, for each $z=(x, 0,0) \in \mathbb{R}^{n+2}$ and each $T>0$ there exists a $k \in \mathbb{N}$ such that $z(t) \in B(0, k)$ for all $a \in \mathcal{A}$ and all $t \in[0, T]$. Furthermore, on $B(0, k)$ the trajectories $z(\cdot)$ and $z_{k}(\cdot)$ coincide and thus we can conclude by $(3.20)$ and by the definition of $\phi$ that

$$
\begin{gathered}
\rho\left(u^{*}(x)\right)=U(x, 0,0) \geq \inf _{a \in \mathcal{A}} \sup _{t \in[0, T]} \phi(z(t)) \\
=\inf _{a \in \mathcal{A}} \sup _{t \in[0, T]} \rho\left((G(x, t, a)-1)+G(x, t, a) \tilde{u}^{*}(\varphi(t))\right) .
\end{gathered}
$$

Using the monotonicity of $\rho$ we obtain

$$
u^{*}(x) \geq \inf _{a \in \mathcal{A}} \sup _{t \in[0, T]}\left\{(G(x, t, a)-1)+G(x, t, a) \tilde{u}^{*}(\varphi(t))\right\}
$$

and hence

$$
u(x) \leq \sup _{a \in \mathcal{A}} \inf _{t \in[0, T]}\{(1-G(x, t, a))+G(x, t, a) \tilde{u}(\varphi(t))\}
$$

holds for each $T \geq 0$, which shows (iii).

Assertion (iv) is proved analogously.

We can now apply these principles to the generalized version of Zubov's equation (3.10).

Proposition 3.6. Let $w$ be a bounded l.s.c. supersolution of (3.10) on $\mathbb{R}^{n}$ with $w(0) \geq 0$. Then $w \geq v$ for $v$ as defined in (3.2).

Proof. First observe that the lower semicontinuity of $w$ and the assumption $w(0) \geq 0$ imply that for each $\epsilon>0$ there exists a $\delta>0$ such that

$$
w(x) \geq-\epsilon \text { for all } x \in \mathbb{R}^{n} \text { with }\|x\| \leq \delta .
$$

Furthermore, the upper optimality principle (3.12) implies

$$
w\left(x_{0}\right) \geq \sup _{a \in \mathcal{A}} \inf _{t \geq 0}\left\{1+G\left(x_{0}, t, a\right)\left(w\left(\varphi\left(t, x_{0}, a\right)\right)-1\right)\right\} .
$$

Now we distinguish two cases:

(i) $x_{0} \in \mathcal{D}_{0}$ : In this case we know that for each $a \in \mathcal{A}$ we have $\varphi\left(t, x_{0}, a\right) \rightarrow 0$ as $t \rightarrow \infty$. Thus from (3.22) and (3.23), and using the definition of $v$ we can conclude

$$
w\left(x_{0}\right) \geq \sup _{a \in \mathcal{A}}\left\{\lim _{t \rightarrow \infty}\left(1-G\left(x_{0}, t, a\right)\right)\right\}=v\left(x_{0}\right) .
$$

which shows the claim.

(ii) $x_{0} \notin \mathcal{D}_{0}$ : In this case by (3.6) and Proposition 3.1(v) it is sufficient to show that 
$w\left(x_{0}\right) \geq 1$. By the definition of $\mathcal{D}_{0}$ we know that for each $T>0$ there exists $a_{T} \in \mathcal{A}$ such that $t\left(x_{0}, a_{T}\right)>T$, which implies $G\left(x_{0}, T, a_{T}\right) \leq \exp \left(-T g_{0}\right)$ which tends to 0 as $T \rightarrow \infty$. Thus denoting the bound on $|w|$ by $M>0$ the inequality (3.23) implies

$$
w\left(x_{0}\right) \geq\left(1-\exp \left(-T g_{0}\right)\right)-\exp \left(-T g_{0}\right) M
$$

for every $T>0$ and hence $w\left(x_{0}\right) \geq 1$.

Proposition 3.7. Let $u$ be a bounded u.s.c. subsolution of (3.10) on $\mathbb{R}^{n}$ with $u(0) \leq 0$. Then $u \leq v$ for $v$ defined in $(3.2)$.

Proof. By the upper semicontinuity of $u$ and $u(0) \leq 0$ we obtain that for every $\epsilon>0$ there exists a $\delta>0$ with $u(x) \leq \epsilon$ for all $x \in \mathbb{R}^{n}$ with $\|x\| \leq \delta$. Thus for each $\epsilon>0$ we find a bounded and continuous function $\tilde{u}_{\epsilon}: \mathbb{R}^{n} \rightarrow \mathbb{R}$ with

$$
\tilde{u}_{\epsilon}(0)<\epsilon \text { and } u \leq \tilde{u}_{\epsilon} \text {. }
$$

Now the lower optimality principle (3.14) implies for every $t \geq 0$ that

$$
u\left(x_{0}\right) \leq \sup _{a \in \mathcal{A}}\left\{1+G\left(x_{0}, t, a\right)\left(\tilde{u}_{\epsilon}\left(\varphi\left(t, x_{0}, a\right)\right)-1\right)\right\}
$$

Again, we distinguish two cases:

(i) $x_{0} \in \mathcal{D}_{0}$ : In this case $\left\|\varphi\left(t, x_{0}, a\right)\right\| \rightarrow 0$ as $t \rightarrow \infty$ uniformly in $a \in \mathcal{A}$. Hence for each $\epsilon>0$ there exists $t_{\epsilon}>0$ such that

$$
\tilde{u}_{\epsilon}\left(\varphi\left(t_{\epsilon}, x_{0}, a\right)\right) \leq \epsilon \quad \text { and } \quad\left|G\left(x_{0}, t_{\epsilon}, a\right)-G\left(x_{0}, \infty, a\right)\right| \leq \epsilon
$$

for all $a \in \mathcal{A}$. Thus from (3.24) and (3.25), and using the definition of $v$ we can conclude

$$
u\left(x_{0}\right) \leq \sup _{a \in \mathcal{A}}\left\{1-(1-\epsilon) G\left(x_{0}, t_{\epsilon}, a\right)\right\} \leq v\left(x_{0}\right)+\epsilon\left(1-v\left(x_{0}\right)\right)+\epsilon,
$$

which shows the claim since $v$ is bounded and $\epsilon>0$ was arbitrary.

(ii) $x_{0} \notin \mathcal{D}_{0}$ : In this case by (3.6) and Proposition 3.1(v) it is sufficient to show that $u\left(x_{0}\right) \leq 1$. By (i) we know that $u(y) \leq v(y)<1$ for each $y \in \mathcal{D}_{0}$, hence analogous to (3.24) for each $\epsilon>0$ we can conclude the existence of a continuous $\tilde{u}_{\epsilon}$ with $u \leq \tilde{u}_{\epsilon}$ and $\tilde{u}_{\epsilon}(y) \leq 1+\epsilon$ for each $y \in \mathcal{D}_{0}$. Since $u$ is bounded by assumption, we may choose $\tilde{u}_{\epsilon}$ such that $M_{\epsilon}:=\sup _{x \in \mathbb{R}^{n}} \tilde{u}_{\epsilon}(x)<\infty$. If $M_{\epsilon} \leq 1$ for some $\epsilon>0$ we are done. Otherwise fix $\epsilon>0$ and consider a sequence $t_{n} \rightarrow \infty$. Then (3.25) implies that there exists a sequence $a_{n} \in \mathcal{A}$ with

$$
u\left(x_{0}\right)-\epsilon \leq 1+G\left(x_{0}, t_{n}, a_{n}\right)\left(\tilde{u}_{\epsilon}\left(\varphi\left(t_{n}, x_{0}, a_{n}\right)\right)-1\right) .
$$

If $\varphi\left(t_{n}, x_{0}, a_{n}\right) \in \mathcal{D}_{0}$ we know that $\tilde{u}_{\epsilon}\left(\varphi\left(t_{n}, x_{0}, a_{n}\right)\right) \leq 1+\epsilon$, and since $G \leq 1$ we obtain $u\left(x_{0}\right)-\epsilon \leq 1+\epsilon$. If $\varphi\left(t_{n}, x_{0}, a_{n}\right) \notin \mathcal{D}_{0}$ then $G\left(x_{0}, t_{n}, a_{n}\right) \leq \exp \left(-g_{0} t_{n}\right)$, thus

$$
1+G\left(x_{0}, t_{n}, a_{n}\right)\left(\tilde{u}_{\epsilon}\left(\varphi\left(t_{n}, x_{0}, a_{n}\right)\right)-1\right) \leq 1+\exp \left(-g_{0} t_{n}\right)\left(M_{\epsilon}-1\right) .
$$

Thus for each $n \in \mathbb{N}$ we obtain

$$
u\left(x_{0}\right) \leq 2 \epsilon+1+\exp \left(-g_{0} t_{n}\right)\left(M_{\epsilon}-1\right),
$$

which for $n \rightarrow \infty$ implies $u\left(x_{0}\right) \leq 1+2 \epsilon$. This proves the assertion since $\epsilon>0$ was arbitrary. 
Using these propositions we can now formulate an existence and uniqueness theorem for the generalized version of Zubov's equation (3.10).

THEOREM 3.8. Consider the system (2.1) and a function $g: \mathbb{R}^{n} \times A \rightarrow \mathbb{R}$ such that (H1) and (H2) are satisfied. Then (3.10) has a unique bounded and continuous viscosity solution $v$ on $\mathbb{R}^{n}$ satisfying $v(0)=0$.

This function coincides with $v$ from (3.2). In particular the characterization $\mathcal{D}_{0}=$ $\left\{x \in \mathbb{R}^{n} \mid v(x)<1\right\}$ holds.

Proof. This is immediate from Propositions 3.6 and 3.7.

For the sake of completeness we state the following analogous result for equation (3.9) which is proved with the same techniques, using (3.13) and (3.15) instead of (3.12) and (3.14). Observe that this result corresponds to the one in [4].

TheOREM 3.9. Consider the system (2.1) and a function $g: \mathbb{R}^{n} \times A \rightarrow \mathbb{R}$. Assume (H1) and (H2). Let $\mathcal{O} \subset \mathbb{R}^{n}$ be an open set containing the origin, and let $U: \mathcal{O} \rightarrow \mathbb{R}$ be a positive and continuous function which is a viscosity solution of (3.9) on $\mathcal{O}$ and satisfies $U(0)=0$ and $U(x) \rightarrow \infty$ for $x \rightarrow \partial \mathcal{O}$ and for $|x| \rightarrow \infty$.

Then $U$ coincides with $V$ from (3.1) and $\mathcal{O}=\mathcal{D}_{0}$. In particular, the function $V$ from (3.1) is the unique positive continuous viscosity solution of equation (3.9) on $\mathcal{D}_{0}$ with $V(0)=0$.

For practical purposes Theorem 3.8 might be inconvenient since we have to compute (or verify) a solution of $(3.10)$ on the whole $\mathbb{R}^{n}$. The following fact can be exploited to show that this is not always necessary.

REMARK 3.2. The optimality principles (i) and (iii) of Proposition 3.5 also hold if we have viscosity sub- or supersolutions of equation (3.10), which are defined only on some proper open subset $\mathcal{O} \subset \mathbb{R}^{n}$, except that in this case the "inf" and "sup" over the time $t$ is only taken up to the first time when the trajectory under consideration leaves $\mathcal{O}$. More precisely, (3.12) becomes

$$
w(x)=\sup _{a \in \mathcal{A}} \sup _{t \in\left[0, \tau_{x}(a)\right]}\{(1-G(x, t, a))+G(x, t, a) w(\varphi(t))\}
$$

and (3.14) becomes

$$
u(x) \leq \sup _{a \in \mathcal{A}} \inf _{t \in\left[0, \tau_{x}(a)\right]}\{(1-G(x, t, a))+G(x, t, a) \tilde{u}(\varphi(t))\} .
$$

where $\tau_{x}(a):=\inf \{t \geq 0 \mid \varphi(t, x, a) \notin \mathcal{O}\}$. We refer to [22] for a proof using the same arguments as in the $\mathbb{R}^{n}$ case combined with a localization technique.

Using these "nonglobal" optimality principles we are now able to state nonglobal versions of the Propositions 3.6 and 3.7.

Proposition 3.10. Consider some open set $\mathcal{O} \subset \mathbb{R}^{n}$. Let $w: \operatorname{cl} \mathcal{O} \rightarrow \mathbb{R}$ be a bounded l.s.c. supersolution of (3.10) on $\mathcal{O}$ with $w(0) \geq 0$ and $w(x) \geq 1$ for all $x \in \partial \mathcal{O}$. Then $w \geq\left. v\right|_{\mathcal{O}}$ for $v$ as defined in (3.2).

Proof. Follows with the same techniques as the proof of Proposition $3.6 \mathrm{using}$ (3.26) instead of (3.12). $\square$

In contrast to Proposition 3.10, we have to strengthen the assumption of Proposition 3.7 in order to get the corresponding nonglobal result.

Proposition 3.11. Consider some open set $\mathcal{O} \subset \mathbb{R}^{n}$. Let $u: \operatorname{cl} \mathcal{O} \rightarrow \mathbb{R}$ be a bounded continuous subsolution of (3.10) on $\mathcal{O}$ with $u(0) \leq 0$ and $u(x)=1$ for all $x \in \partial \mathcal{O}$. Then $u \leq\left. v\right|_{\mathcal{O}}$ for $v$ as defined in (3.2).

Proof. It is sufficient to show that $\mathcal{D}_{0} \subseteq \mathcal{O}$ since in this case we get $\left.v\right|_{\partial \mathcal{O}} \equiv 1$ and thus obtain the assertion with the same techniques as in the proof of Proposition 3.7 using (3.27) instead of (3.14). 
In order to show $\mathcal{D}_{0} \subseteq \mathcal{O}$ assume that $\mathcal{D}_{0} \nsubseteq \subseteq \mathcal{O}$. Then we obtain

$$
r_{0}:=\sup \left\{r>0 \mid\left\{x \in \mathbb{R}^{n} \mid v(x) \leq r\right\} \subset \mathcal{O}\right\}<1 .
$$

We set $S:=\left\{x \in \mathbb{R}^{n} \mid v(x) \leq r_{0}\right\}$. Note that from the optimality principle (3.4) we immediately obtain that $v$ is strictly decreasing along each trajectory $\varphi\left(t, x_{0}, a\right)$, hence $\varphi\left(t, x_{0}, a\right) \in \operatorname{int} S \subseteq \mathcal{O}$ for all $t>0, a \in \mathcal{A}$. By definition of $r_{0}$ there exists $x_{0} \in \partial \mathcal{O}$ with $v\left(x_{0}\right)=r_{0}$ and $u\left(x_{0}\right)=1$, hence by continuity of $u$ there exists $\epsilon>0$ and $\eta>0$ such that $u(x)>r_{0}+\epsilon$ for all $x \in \mathcal{O} \cap B\left(x_{0}, \eta\right)$. Fixing some arbitrary $a^{*} \in \mathcal{A}$ and some $\tau>0$ sufficiently small we set $x_{1}:=\varphi\left(\tau, x_{0}, a^{*}\right) \in \mathcal{O} \cap B\left(x_{0}, \eta\right)$. Then $\varphi\left(t, x_{1}, a\right) \in \operatorname{int} S \subseteq \mathcal{O}$ for all $t \geq 0, a \in \mathcal{A}$, i.e. the trajectory never reaches $\partial \mathcal{O}$ implying that (3.27) coincides with (3.14) (note that by continuity of $u$ we can choose $\tilde{u}=u)$. Thus we obtain

$$
r_{0}+\epsilon \leq u\left(x_{1}\right) \leq \sup _{a \in \mathcal{A}} \inf _{t \in[0, T]}\left\{(1-G(x, t, a))+G(x, t, a) u\left(\varphi\left(t, x_{1}, a\right)\right)\right\}
$$

for all $T>0$. Since $u$ is continuous with $u(0) \leq 0$ and $\varphi\left(t, x_{1}, a\right) \rightarrow 0$ as $t \rightarrow \infty$ we obtain by letting $T \rightarrow \infty$

$$
r_{0}+\epsilon \leq u\left(x_{1}\right) \leq \lim _{t \rightarrow \infty} \sup _{a \in \mathcal{A}}\{(1-G(x, t, a))\}=v\left(x_{1}\right) \leq r_{0}
$$

which is a contradiction and hence shows $\mathcal{D}_{0} \subseteq \mathcal{O}$.

From these propositions we can now easily deduce the following theorem. It shows that we can restrict ourselves to a proper open subset $\mathcal{O}$ of the state space and still obtain our solution $v$, provided $\mathcal{D}_{0} \subseteq \mathcal{O}$. Conversely, if $\mathcal{D}_{0} \nsubseteq \mathcal{O}$ then no viscosity solution $v$ with $v(x)=1$ for all $x \in \partial \mathcal{O}$ can exist.

THEOREM 3.12. Consider the system (2.1) and a function $g: \mathbb{R}^{n} \times A \rightarrow \mathbb{R}$. Assume (H1) and (H2). Let $\mathcal{O} \subset \mathbb{R}^{n}$ be an open set containing the origin, and let $v: \operatorname{clO} \rightarrow \mathbb{R}$ be a bounded and continuous function which is a viscosity solution of (3.10) on $\mathcal{O}$ and satisfies $v(0)=0$ and $v(x)=1$ for all $x \in \partial \mathcal{O}$.

Then $v$ coincides with the restriction $\left.v\right|_{\mathcal{O}}$ of the function $v$ from (3.2). In particular the characterization $\mathcal{D}_{0}=\{x \in \mathcal{O} \mid v(x)<1\}$ holds.

Proof. Follows immediately from the Propositions 3.10 and 3.11.

4. Further properties of the solution. In this section we collect several properties of the solution $v$ of Zubov's equation from Theorem 3.8. In particular we show that this solution is a robust Lyapunov function on $\mathcal{D}_{0}$, and that additional assumptions on $g$ ensure Lipschitz continuity of $v$.

THEOREM 4.1. The function $v$ is a robust Lyapunov function for the system (2.1). More precisely we have

$v\left(\varphi\left(t, x_{0}, a(\cdot)\right)\right)-v\left(x_{0}\right) \leq\left[1-\exp \left(-\int_{0}^{t} g(\varphi(\tau), a(\tau)) d \tau\right)\right]\left(v\left(\varphi\left(t, x_{0}, a(\cdot)\right)\right)-1\right)<0$ for all $x_{0} \in \mathcal{D}_{0} \backslash\{0\}$ and all $a(\cdot) \in \mathcal{A}$. In particular each sublevel set of $v$ is positively invariant.

Proof. The dynamic programming principle (3.4) implies

$$
v(x) \geq 1-\exp \left(\int_{0}^{t} g(\varphi(\tau, x, a), a(\tau)) d \tau\right)+\exp \left(\int_{0}^{t} g(\varphi(\tau, x, a), a(\tau)) d \tau\right) v(\varphi(t, x, a))
$$

for each $a \in \mathcal{A}$. This immediately yields the assertion.

REMARK 4.1. 
(i) If $v$ is differentiable in some point $0 \neq x_{0} \in \mathcal{D}_{0}$ this yields the more familiar inequality

$$
\sup _{a \in A} D v\left(x_{0}\right) f(x, a) \leq\left(v\left(x_{0}\right)-1\right) g(x, a)<0
$$

which, in fact, can also be directly derived from (3.10).

(ii) It follows immediately from Proposition 3.5 (ii) that any viscosity supersolution $w$ of $(3.10)$ with $w(0)=0$ is a robust Lyapunov function on its sublevel set $\left\{x \in \mathbb{R}^{d} \mid w(x)<1\right\}$.

Now we investigate regularity properties for the function $v$. In general, we cannot expect this function to be differentiable. A suitable choice of $g$, however, guarantees Lipschitz continuity. We start by investigating this for the function $V$ defined in (3.1).

Proposition 4.2. Assume (H1) and (H2) and that $f(\cdot, a)$ is locally Lipschitz continuous uniformly in a, i.e. for any $R>0$ there exists a constant $M_{R}$ such that

$$
\|f(x, a)-f(y, a)\| \leq M_{R}\|x-y\|, \quad \text { for all } x, y \in B(0, R), a \in A .
$$

Assume furthermore that there exists a neighborhood $N$ of the origin such that for all $x, y \in N$ the inequality

$$
|g(x, a)-g(y, a)| \leq K \max \{\|x\|,\|y\|\}^{s}\|x-y\|
$$

holds for some $K>0$ and $s>M_{r} / \sigma$ with $r>0, \sigma>0$ as in (H1). Then $V$ is locally Lipschitz in $\mathcal{D}_{0}$.

Proof. Let $S \subset \mathcal{D}_{0}$ be compact. According to (H2) there exists a time $T>0$ such that $\varphi(t, x, a) \in N \cap B(0, r)$ for all $t \geq T, x \in S, a \in \mathcal{A}$. Furthermore, the set $\mathcal{R}(S, T)$ is bounded and we may choose $R>0$ large enough so that $\mathcal{R}(S, T) \subset B(0, R)$. Now fix $x, y \in S$. Analogous to the proof of Proposition 3.1(iii) we obtain

$$
\begin{aligned}
|V(x)-V(y)| \leq & \sup _{a \in \mathcal{A}} \int_{0}^{+\infty}|g(\varphi(t, x, a), a(t))-g(\varphi(t, y, a), a(t))| d t \\
\leq & \sup _{a \in \mathcal{A}} \int_{0}^{T}|g(\varphi(t, x, a), a(t))-g(\varphi(t, y, a), a(t))| d t \\
& +\sup _{a \in \mathcal{A}} \int_{T}^{+\infty}|g(\varphi(t, x, a), a(t))-g(\varphi(t, y, a), a(t))| d t \\
\leq & \int_{0}^{T} L_{R} e^{M_{R} t}\|x-y\| d t \\
& +\int_{T}^{+\infty} K_{\max \{\|\varphi(T)\|,\|y(T)\|\}^{s} C^{s} e^{-s \sigma(t-T)} e^{M_{r} t}\|x-y\| d t}^{\leq} \\
& \underbrace{\left(L_{R} \frac{e^{M_{R} T}-1}{M_{R}}+K r^{s} C^{s} e^{\sigma T} \frac{e^{\left(M_{r}-s \sigma\right) T}}{s \sigma-M_{r}}\right)}_{=L_{S}}\|x-y\| .
\end{aligned}
$$

This shows the assertion. $\square$

Obviously, this result immediately carries over to $v$ on $\mathcal{D}_{0}$. In order to obtain Lipschitz continuity of $v$ on the rest of $\mathbb{R}^{n}$ it is convenient to consider a generalization of the transformation (3.2) by defining

$$
v_{\delta}(x):=1-\exp (-\delta V(x))
$$


for $\delta>0$. Observe that this results in the equation

$$
\inf _{a \in A}\{-f(x, a) D v(x)-\delta(1-v(x)) g(x, a)\}=0 \quad x \in \mathbb{R}^{n} .
$$

Thus this transformation is equivalent to an appropriate choice of $g$ in (3.10). Observe that for $\delta \rightarrow 0$ the function $v_{\delta}$ converges to 0 on $\mathcal{D}_{0}$ and is equal to 1 outside $\mathcal{D}_{0}$. Note that this convergence to a piecewise constant function is a typical behavior of discounted optimal value functions, see, e.g., [9].

In the opposite case, i.e. for sufficiently large $\delta>0$ the following result holds for $v_{\delta}$.

Proposition 4.3. Assume that $f(\cdot, a)$ and $g(\cdot, a)$ are globally Lipschitz continuous in $\mathbb{R}^{n}$, with constants $L_{f}, L_{g}>0$ independent of $a \in A$, and assume that there exists a neighborhood $N$ of the origin such that for all $x, y \in N$ the inequality

$$
|g(x, a)-g(y, a)| \leq K \max \{\|x\|,\|y\|\}^{s}\|x-y\|
$$

holds for some $K>0$ and $s>L_{f} / \sigma$ with $\sigma>0$ given by (H1). Then the function $v_{\delta}$ is Lipschitz continuous in $\mathbb{R}^{n}$ for all $\delta>0$ sufficiently large.

Proof. Let $L_{0}$ denote the Lipschitz constant of $V$ on $B(0, r)$ guaranteed by Proposition 4.2. For $x \in \mathcal{D}_{0}$, define $T_{x}=\sup \{t(x, a): a \in \mathcal{A}\}$ and observe that $V(x) \geq g_{0} T_{x}$, where $g_{0}>0$ is given by (H2). If $x, y \in \mathcal{D}_{0}$, then for any $\epsilon>0$, there exists a control $a \in \mathcal{A}$ such that

$$
\begin{aligned}
|V(x)-V(y)| \leq & \int_{0}^{T_{x} \vee T_{y}}|g(\varphi(t, x, a), a(t))-g(\varphi(t, y, a), a(t))| d t+ \\
& +\left|V\left(\varphi\left(T_{x} \vee T_{y}, x, a\right)\right)-V\left(\varphi\left(T_{x} \vee T_{y}, y, a\right)\right)\right|+\epsilon \\
& \leq \int_{0}^{T_{x} \vee T_{y}} L_{g} \exp \left(L_{f} t\right)\|x-y\| d t+L_{0}\|x-y\| \exp \left(L_{f}\left(T_{x} \vee T_{y}\right)\right)+\epsilon \\
& \leq\left(L_{0}+L_{g} / L_{f}\right) \exp \left(L_{f} V(x) / g_{0}\right)\|x-y\|+\epsilon .
\end{aligned}
$$

So we see that $V$ is locally Lipschitz continuous in $\mathcal{D}_{0}$ with a constant of the form $L \exp \left(L_{f} V(x) / g_{0}\right)$.

Let $\phi \in C^{1}\left(\mathbb{R}^{n}\right)$ be such that $v_{\delta}(x)-\phi$ has a local maximum at $x_{0} \in \mathcal{D}_{0}$, where we may assume that $v_{\delta}\left(x_{0}\right)-\phi\left(x_{0}\right)=0$ and $\phi(x) \leq 1, \forall x \in \mathbb{R}^{n}$. Then $V-\psi$ has a local maximum at $x_{0}$ for $\psi(x)=-\ln (1-\phi(x)) / \delta$.

It follows that

$$
\left|D \phi\left(x_{0}\right)\right| \leq \delta\left|D \psi\left(x_{0}\right)\right| \exp (-\delta V(x)) \leq L \delta \exp \left(\left(L_{f} / g_{0}-\delta\right) V(x)\right) .
$$

Hence, letting $\delta \geq L_{f} / g_{0}$ and recalling that $v_{\delta} \equiv 1$ in $\mathbb{R}^{n} \backslash \mathcal{D}_{0}$, we have that $\left|D \phi\left(x_{0}\right)\right| \leq$ $\delta L$ for any $x \in \mathbb{R}^{n}$ and for any $\phi \in C^{1}\left(\mathbb{R}^{n}\right)$ such that $v_{\delta}(x)-\phi$ has a local maximum at $x$. This implies that $v_{\delta}$ is Lipschitz continuous in $\mathbb{R}^{n}$ with Lipschitz constant $\delta L$, cp. [5, Lemma 2.10].

5. Smooth Solutions. It is always of interest to know whether for a given stability property there are Lyapunov functions with certain regularity properties. In [15] it is shown that under the condition of global uniform asymptotic stability; that is, under the condition $\mathcal{D}_{0}=\mathbb{R}^{n}$ in our terminology, there exists a $C^{\infty}$ Lyapunov function $V: \mathbb{R}^{n} \rightarrow \mathbb{R}$ such that

$$
D V(x) f(x, a) \leq-\alpha_{1}(\|x\|)
$$


for some class $\mathcal{K}_{\infty}$ function $\alpha_{1}$. Furthermore, there exist class $\mathcal{K}_{\infty}$ functions $\alpha_{2}, \alpha_{3}$ such that

$$
\alpha_{2}(\|x\|) \leq V(x) \leq \alpha_{3}(\|x\|)
$$

(as usual in stability theory we call a function $\alpha:[0, \infty) \rightarrow[0, \infty)$ of class $\mathcal{K}_{\infty}$ if it is continuous, strictly increasing, unbounded and satisfies $\alpha(0)=0)$. By [19, Theorems $1 \& 2$, Proposition 3] it follows that if we add the assumption that $f(x, A)$ be convex for all $x \in \mathbb{R}^{n}$ then there exists a $C^{\infty}$ Lyapunov function $V$ on $\mathcal{D}_{0}$ (which is in this case equal to $\mathcal{D}$ by Proposition 2.3 (iii)). Assuming that $\omega: \mathcal{D} \rightarrow \mathbb{R}_{\geq 0}$ is continuous, and satisfies $\omega(x)=0$ if and only if $x=0$, and $\omega\left(x_{n}\right) \rightarrow \infty$ for any sequence $\left\{x_{n}\right\}$ with $\lim x_{n} \in \partial \mathcal{D}$ or $\lim \left\|x_{n}\right\|=\infty$, then $V$ can be chosen in such a manner, that it has the properties (5.1) $(5.2)$, where $\|x\|$ has to be replaced by $\omega(x)$. It is of interest, and therefore the topic of our last section, to know whether we are able to reproduce these functions via our approach.

We first treat the case of global stability.

LEMMA 5.1. Assume that system (2.1) is globally uniformly asymptotically stable at 0 then $g(x, a)$ can be chosen such that the corresponding solutions $V$ of (3.9) and $v$ of (3.10) are $C^{\infty}$. Furthermore, for any smooth Lyapunov function $V$ satisfying (5.1) and (5.2) there exists a function $g: \mathbb{R}^{n} \rightarrow \mathbb{R}$ such that $V$ is the corresponding solution of $(3.9)$.

Proof. By [15, Theorem 2.9, Remark 4.1] there exists a $C^{\infty}$ Lyapunov function $V: \mathbb{R}^{n} \rightarrow \mathbb{R}$ for $(2.1)$. Now define $v(x)=1-e^{-V(x)}$ as before and

$$
\begin{array}{r}
g(x, a):=g(x):=-\sup _{a \in \mathcal{A}} \frac{D v(x) f(x, a)}{1-v(x)}= \\
-\sup _{a \in \mathcal{A}} \frac{e^{-V(x)} D V(x) f(x, a)}{e^{-V(x)}}=-\sup _{a \in A} D V(x) f(x, a) .
\end{array}
$$

It is clear that the function $g$ thus defined satisfies condition (i) of (H2). By (5.1) we have $g(x) \geq \alpha_{1}(\|x\|)$ which implies (ii). The third condition condition is implied by Lipschitz continuity of $f$ and smoothness of $V$. A straightforward computation yields that $V, v$ are the respective (unique) solutions of (3.9) and (3.10).

The second statement is clear by the previous construction. $\square$

It is now tempting to try to copy this argument for the non global case by utilizing the smooth maximal Lyapunov functions defined on the domain of attraction which are obtained in [19]. In this way one might hope to construct smooth Lyapunov functions that are representable as suitable solutions of (3.9), respectively (3.10). This approach however has one problem: It is by no means clear that $g$ as defined in the proof of Lemma 5.1 can be continuously extended to $\mathbb{R}^{n}$ so that (H2) is satisfied. We can, however, reconstruct smooth solutions on any subset of $\mathcal{D}_{0}$ that is bounded away from $\partial \mathcal{D}_{0}$.

Proposition 5.2. Assume (H1), (H2) and that $f(x, A)$ is convex for all $x \in \mathbb{R}^{n}$. Let $B \subset \mathcal{D}_{0}$ satisfy $\operatorname{dist}\left(B, \partial \mathcal{D}_{0}\right)>0$, then there exists a function $g: \mathbb{R}^{n} \rightarrow \mathbb{R}$ such that the corresponding solution $v$ of (3.10) is $C^{\infty}$ on a neighborhood of $B$.

Proof. Let $V$ denote a smooth Lyapunov function for system (2.1) defined on $\mathcal{D}$. Let $U$ be an open neighborhood of $B$ contained in $\mathcal{D}_{0}$ and define $g_{\mid U}$ by (5.3). Then $g$ can be extended to a continuous function on $\mathbb{R}^{n}$ satisfying (H2). The corresponding unique solution $v$ of $(3.10)$ is $C^{\infty}$ on $U$. 
6. Example. In this section we illustrate our results by a simple example where we explicitly verify a (nonsmooth) solution of the generalized version of Zubov's equation (3.10). Consider the system

$$
\begin{aligned}
& \dot{x}_{1}=-x_{1}+a x_{1}^{2} \\
& \dot{x}_{2}=-x_{2}+a x_{2}^{2}
\end{aligned}
$$

with $x=\left(x_{1}, x_{2}\right)^{T} \in \mathbb{R}^{2}$ and $A=[-1,1]$. We claim that for $g(x, a)=\|x\|^{2}=x_{1}^{2}+x_{2}^{2}$ the function $v$ defined by

$$
v(x)= \begin{cases}1-e^{-V(x)}, & x \in(-1,1)^{2} \\ 1, & x \notin(-1,1)^{2}\end{cases}
$$

where $V:(-1,1)^{2} \rightarrow \mathbb{R}$ is given by

$$
V(x)= \begin{cases}-\ln \left(1-x_{1}\right)-\ln \left(1-x_{2}\right)-x_{1}-x_{2}, & x_{1} \geq-x_{2} \\ -\ln \left(1+x_{1}\right)-\ln \left(1+x_{2}\right)+x_{1}+x_{2}, & x_{1} \leq-x_{2}\end{cases}
$$

solves equation (3.10).

Note that by Theorem 3.12 it suffices to verify the equation $(3.10)$ on $(-1,1)^{2}$, since $\left.v\right|_{(-1,1)^{2}}$ satisfies the assumptions of this theorem with $\mathcal{O}=(-1,1)^{2}$.

Using Remark 3.1 we identify the set of possible derivatives of functions $\phi$ such that $v-\phi$ has a local extremum for $x \in(-1,1)$. First note that $v$ is smooth on $(-1,1)^{2} \backslash D_{1}$ where $D_{1}$ is the diagonal $\left\{x \in(-1,1)^{2} \mid x_{1}=-x_{2}\right\}$. In this region $D \phi$ must coincide with $D v$ which is computed to be

$$
D v(x)= \begin{cases}\left(x_{1}\left(1-x_{2}\right) e^{+x_{1}+x_{2}}, x_{2}\left(1-x_{1}\right) e^{+x_{1}+x_{2}}\right), & x \in(-1,1)^{2}, x_{1}>-x_{2} \\ \left(x_{1}\left(1+x_{2}\right) e^{-x_{1}-x_{2}}, x_{2}\left(1+x_{1}\right) e^{-x_{1}-x_{2}}\right), & x \in(-1,1)^{2}, x_{1}<-x_{2} .\end{cases}
$$

On $D_{1}$ (setting $x=(y,-y)^{T}$ ) one verifies that the superdifferential $D^{+} v$ is empty while the subdifferential $D^{-} v$ satisfies

$$
D^{-} v(y,-y)=\left\{\theta p_{1}+(1-\theta) p_{2} \mid \theta \in[0,1]\right\}
$$

where

$$
\begin{aligned}
& p_{1}=(+y(y+1),+y(y-1)) \\
& p_{2}=(-y(y-1),-y(y+1)) .
\end{aligned}
$$

Using these computations we obtain that on $(-1,1)^{2}$ equation $(3.10)$ becomes

$$
\begin{array}{cc}
\min _{a \in[-1,1]}\left\{-e^{x_{1}+x_{2}}(1-a)\left(x_{1}^{3}+x_{2}^{3}-x_{1} x_{2}^{3}-x_{1}^{3} x_{2}\right)\right\}=0 & \text { for } x_{1}>-x_{2}, \\
\min _{a \in[-1,1]}\left\{e^{-x_{1}-x_{2}}(1+a)\left(x_{1}^{3}+x_{2}^{3}+x_{1}^{3} x_{2}+x_{1} x_{2}^{3}\right)\right\}=0 & \text { for } x_{1}<-x_{2}
\end{array}
$$

and

$$
\left.\min _{a \in[-1,1]}\left\{2(1-a+2 \theta a) y^{4}\right)\right\} \geq 0 \quad \text { for } x_{1}=-x_{2}=: y .
$$

It turns out that in the first case the minimizer is $a=1$, in the second case it is $a=-1$, while in the third case it is $a=1$ for $\theta \in[0,1 / 2), a=-1$ for $\theta \in(1 / 2,1]$ and any $a \in[-1,1]$ for $\theta=1 / 2$. In all cases we see that the desired (in)equalities are satisfied, which in particular shows that $\mathcal{D}_{0}=(-1,1)^{2}$.

Acknowledgment: The authors would like to thank Pierpaolo Soravia and Maurizio Falcone for useful discussions. 


\section{REFERENCES}

[1] M. Abu Hassan and C. Storey, Numerical determination of domains of attraction for electrical power systems using the method of Zubov. Int. J. Control 34 (1981), 371-381.

[2] B. Aulbach, Asymptotic stability regions via extensions of Zubov's method. I and II. Nonlinear Anal., Theory Methods Appl. 7 (1983), 1431-1440 and 1441-1454.

[3] M. Bardi and I. Capuzzo Dolcetta, Optimal Control and Viscosity Solutions of HamiltonJacobi-Bellman equations, Birkhäuser, Boston, 1997.

[4] M. Bardi and P. Soravia, Hamilton-Jacobi equations with singular boundary conditions on a free boundary and applications to differential games, Trans. Amer. Math. Soc. 325 (1991), 205-229.

[5] G. Barles, Solutions de viscosité des equations de Hamilton-Jacobi, Springer-Verlag, Paris, 1994.

[6] N. Bhatia. On asymptotic stability in dynamical systems. Math. Syst. Theory 1 (1967), 113127.

[7] F. Camilli, L. Grüne, and F. Wirth. A regularization of Zubov's equation for robust domains of attraction. In: Nonlinear Control in the Year 2000, A. Isidori et. al. eds., Lecture Notes in Control and Information Sciences, Springer-Verlag, London, 2000, to appear.

[8] C. Coleman. Local trajectory equivalence of differential systems. Proc. Amer. Math. Soc. 16 (1965), 890-892. Addendum. Ibid. 17 (1966), 770.

[9] L. Grüne, On the relation between discounted and average optimal control problems, J. Differ. Equations 148 (1998), 65-99.

[10] W. Hahn, Stability of Motion, Springer-Verlag, Berlin, 1967.

[11] V. Jurdjevic. Geometric Control Theory. Cambridge University Press, 1997.

[12] N.E. Kirin, R.A. Nelepin, and V.N. Bajdaev, Construction of the attraction region by Zubov's method. Differ. Equations 17 (1982), 871-880.

[13] H. K. Khalil. Nonlinear Systems. 2nd ed. Prentice-Hall, 1996.

[14] E. B. Lee and L. Markus. Foundations of Optimal Control Theory. John Wiley \& Sons, New York, 1967.

[15] Y. Lin, E. D. Sontag, and Y. Wang. A smooth converse Lyapunov theorem for robust stability. SIAM J. Control Optim., 34 (1996), 124-160.

[16] J.W. Milnor. Differential topology. in Lectures in Modern Mathematics II. John Wiley \& Sons, New York, 165-183, 1964.

[17] A.D.B. Paice and F. Wirth. Robustness analysis of domains of attraction of nonlinear systems, Proceedings of the Mathematical Theory of Networks and Systems MTNS98, pages $353-$ 356, Padova, Italy, 1998.

[18] A.D.B. Paice and F. Wirth. Robustness of nonlinear systems subject to time-varying perturbations, In F. Colonius et al. (eds.), Advances in Mathematical Systems Theory, Birkhäuser, Boston, 2000. To appear.

[19] L. Praly and A. Teel. A smooth Lyapunov function from a class- $\mathcal{K} \mathcal{L}$ estimate involving two positive semidefinite functions. Control, Optimization and Calculus of Variations, 2000. to appear.

[20] E. D. Sontag and Y. Wang. New characterizations of input to state stability IEEE Trans. Autom. Control, 41 (1996): 1283-1294.

[21] P. Soravia, Optimality principles and representation formulas for viscosity solutions of Hamilton-Jacobi equations, I: Equations of unbounded and degenerate control problems without uniqueness, Advances Diff. Equations, 4 (1999), 275-296.

[22] P. Soravia, Optimality principles and representation formulas for viscosity solutions of Hamilton-Jacobi equations, II: Equations of control problems with state constraints, Diff. Integr. Equations, 12 (1999), 275-293.

[23] A. Vannelli and M. Vidyasagar. Maximal Lyapunov functions and domains of attraction for autonomous nonlinear systems. Automatica, 21 (1985), 69-80.

[24] F.W. Wilson. The structure of the level surfaces of a Lyapunov function. J. Differ. Equations 3 (1967), 323-329.

[25] V.I. Zubov, Methods of A.M. Lyapunov and their Application, P. Noordhoff, Groningen, 1964. 